\title{
Magnetic Particle-Scanning for Ultrasensitive
}

\section{Immunodetection on-Chip}

Matteo Cornaglia, Raphaël Trouillon, H. Cumhur Tekin`, Thomas Lehnert, and Martin A. M. Gijs*

Laboratory of Microsystems, Ecole Polytechnique Fédérale de Lausanne, CH-1015 Lausanne, Switzerland

* Author to whom correspondence should be addressed. E-mail: martin.gijs@epfl.ch

KEYWORDS. Surface-based immunoassays, protein detection, superparamagnetic particles, magnetic particle-scanning, random-walk model

ABSTRACT. We describe the concept of magnetic particle-scanning for on-chip detection of biomolecules: a magnetic particle, carrying a low number of antigens (Ags) (down to a single molecule), is transported by hydrodynamic forces and is subjected to successive stochastic reorientations in an engineered magnetic energy landscape. The latter consists of a pattern of substrate-bound small magnetic particles that are functionalized with antibodies (Abs). Subsequent counting of the captured antigen-carrying particles provides the detection signal. The magnetic particle-scanning principle is investigated in a custom-built magneto-microfluidic chip and theoretically described by a random walk-based model, in which the trajectory of the contact point between an antigen-carrying particle and the small magnetic particle pattern is described by 
stochastic moves over the surface of the mobile particle, until this point coincides with the position of an Ag resulting in the binding of the particle. This model explains the particular behavior of previously reported experimental dose-response curves obtained for two different ligand-receptor systems (biotin/streptavidin and TNF- $\alpha$ ) over a wide range of concentrations. Our model shows that magnetic particle-scanning results in a very high probability of immunocomplex formation for very low antigen concentrations leading to an extremely low limit of detection, down to the single molecule-per-particle level. When compared to other types of magnetic particle-based surface coverage assays, our strategy was found to offer a wider dynamic range $(>8$ orders of magnitude), as the system does not saturate for concentrations as high as $10^{11} \mathrm{Ag}$ molecules in a 5 $\mu l$ drop. Furthermore, by emphasizing the importance of maximizing the encounter probability between the $\mathrm{Ag}$ and the $\mathrm{Ab}$ to improve sensitivity, our model also contributes at explaining the behavior of other particle-based heterogeneous immunoassays.

Immunoassays are essential in clinical diagnostics to differentiate between health and disease and for therapeutic monitoring. A major goal is disease detection at an early stage to minimize the invasiveness of the treatment and maximize the chance of healing. ${ }^{1,2}$ To reach this goal, the measurement of extremely low concentrations of proteins in serum is necessary. ${ }^{3-7}$ At the same time, point-of-care medical diagnostics represents one of today's main challenges towards the promise of tests that can be carried out at the site of patient care. ${ }^{8}$ From the immunoassay integration perspective, this has been translated in terms of miniaturization of the detection systems and reduction of the sample and reagent volumes needed for the analysis. The conjunction of these factors is dictating the need of a new generation of fast, cheap and minimally-invasive diagnostics tools, able to selectively detect dramatically low amounts of proteins - down to the limit of a single 
molecule - in complex media. For instance, clinically relevant concentration values of tumor necrosis factor $\alpha$ (TNF- $\alpha$ ) or interleukins (e.g. IL-6) for early disease detection are in the femtomolar regime or lower $\left(<10^{-15} \mathrm{M}\right){ }^{9}$ A minimally invasive immunoassay should ideally be performed starting from a sample volume the size of a blood droplet (typically 5 to $10 \mu \mathrm{L}$ ), thus containing at maximum a few thousand target antigens (Ags). Recent technological advances, in particular those enabled by microfluidics, have therefore been creating novel tools for detecting biomarkers at low concentrations and/or sample volumes in complex sample media and with a wide variety of assay read-outs. ${ }^{10-18}$ Functionalized superparamagnetic particles have been shown to be ideally suited for the rapid and efficient capture and isolation of target molecules. ${ }^{19,20}$ Many of these magneto-microfluidic assays were based on a protocol, in which magnetic particles were immobilized in a flow and exposed to a series of sequential reagent exposure and washing steps, as inspired by a batch-type assay ${ }^{21-23}$, but also continuous flow microfluidic assays have been proposed, in which magnetic particles were moved through multilaminar reagent/washing solution flow streams to implement the assay protocol. ${ }^{24}$ Also droplet-based systems have been proposed that allowed storage of reagent/washing solutions in aqueous droplets surrounded by an immiscible oil phase and employed magnetic particles that were moved between the different droplets to perform the assay protocol. ${ }^{25,26}$ When assaying samples having an extremely low analyte concentration, the ratio between the amount of target molecules and the amount of particles used for their capture is usually very large. As a result, the percentage of particles carrying analyte molecules follows a Poisson distribution - i.e. particles carry either a single molecule or none. In this regime, quantification of the amount of molecule-carrying particles is based on single analyte molecule detection events. ${ }^{27}$ However, the size mismatch between target molecules and particles may complicate the detection. For instance, the typical size of molecules of interest being in the 
nanometer range (the Stokes radius of a TNF- $\alpha$ monomer was estimated as $2.2 \mathrm{~nm}$ at $\mathrm{pH}=7.0)^{28}$, a captured molecule occupies only $\sim 10^{-7}$ of the surface of a $300 \mathrm{~nm}$ diameter particle. This ratio drops to $\sim 10^{-9}$ and $\sim 10^{-11}$ for $3 \mu \mathrm{m}$ and $30 \mu \mathrm{m}$ particles, respectively. If we intend to capture these particles on a substrate aiming to count them for single molecule immunodetection, these values are indicative of the - extremely low - probability of encounter between a particle-linked Ag and substrate-immobilized antibodies (Abs). Since in such case both single Ag molecules (ligands) and capture Abs (receptors) are bound to surfaces, their recognition kinetics is substantially reduced as compared to freely diffusing species; therefore the formation of specific immunocomplexes would be very slow or probabilistically almost impossible. ${ }^{29}$ However, solutions to this problem are offered on the nanoscale. A first option could be reducing the particles' size, ideally down to a few tens of nm, to reduce the size-mismatch. Although this would improve ligand-receptor encounter probabilities, it would result in much more challenging particle manipulation, capture and detection. In order to take advantage of the size of micrometer particles, an alternative solution is increasing the rotational diffusion of the Ag-carrying particles to enhance the Ag-Ab encounter probability. While this concept has been proposed for agglutination immunoassays in solution ${ }^{29,30}$, it is still unexplored in surface-based assays.

Here, we describe the concept of magnetic particle-scanning, which was used in a previous report ${ }^{18}$ to significantly increase the probability of immunocomplex formation in single molecule-based heterogeneous immunoassays. However, the enhancement mechanism was not described then, as we chose to focus on the detection capabilities of our immunoassay. Therefore, in this new paper, we introduce the physics of this concept, in which a particle carrying a single Ag is subjected to successive stochastic reorientations in an engineered magnetic energy landscape, as established by a regular pattern of magnetic particles, while transported by fine-tuned hydrodynamic forces in a 
carefully optimized microfluidic system. In this mechanism, as the moving particle slides over the immobilized ones, the point of contact between the two particles continuously explores, or "scans", a section of the particle surface. If this inter-particle interaction is repeated several times, a large fraction of the moving bead can be interrogated, until the presence of a bound Ag triggers the particle binding. We explore this phenomenon in a custom-built magneto-microfluidic chip. Finally we introduce a random walk-based model for describing the magnetic particle-scanning process, explaining the particular dose-response curves of previously reported protein detection experiments over an extremely large concentration range. ${ }^{18}$ Moreover, our analysis provides understanding of the features of other particle-based heterogeneous immunoassays. 


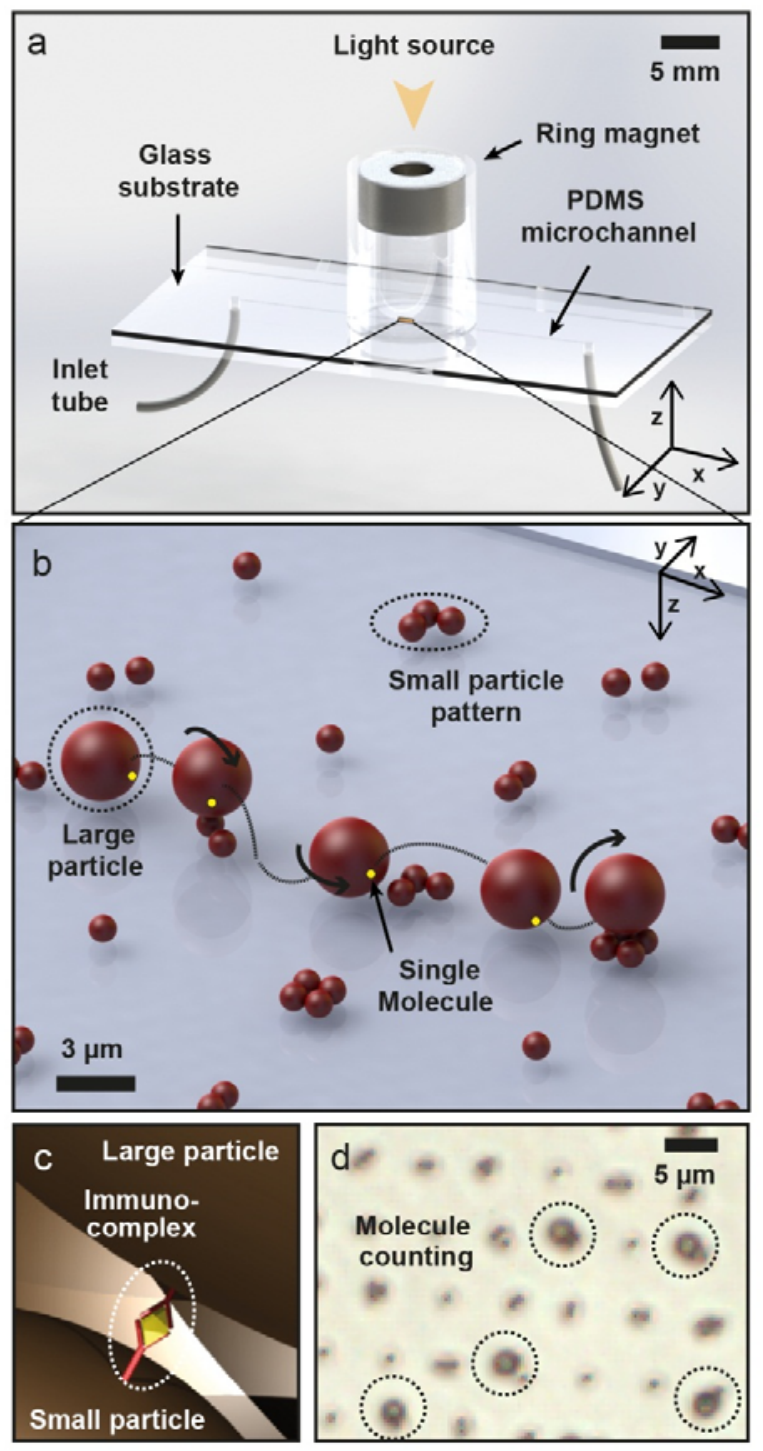

Figure 1. (a) Schematic representation of the magneto-microfluidic device used for investigating the "magnetic particle-scanning" mechanism. (b) Time sequence of a large particle sliding over the substrate and interacting with the functionalized surface on the small particles. (c) Specific immunocomplex between a large and a small particle due to the presence of a single Ag on the large particle. (d) Counting of the number of captured large particles yields the Ag-concentration. 


\section{EXPERIMENTAL METHODS}

Single-molecule detection principle. A magneto-microfluidic system, shown in Figure 1a, has been employed to observe and study the magnetic particle-scanning mechanism for ultrasensitive protein detection. Functionalized "small" (1 $\mu \mathrm{m}$-diameter) superparamagnetic particles are patterned on a glass substrate, which is clamped to a polydimethysiloxane (PDMS) halfmicrochannel. A constant vertical field at the glass substrate, as induced by a permanent magnet placed above the chip, imposes a constant magnetic moment to the immobilized particles. The magnet ring shape ensures efficient light exposure to the immobilized particles, for accurate timelapse observation of the magnetic particles via a high-speed camera. The magnet and small particles provide to the substrate a locally engineered magnetic energy landscape, in which functionalized "large" (2.8 $\mu \mathrm{m}$-diameter) magnetic particles, which have previously captured target Ags from a matrix, are transported at a controlled flow rate. Under optimized magnetic and hydrodynamic drag force conditions, the large particles gently slide over the substrate, slow down at the positions of the small particles and interact with the functionalized surface of the latter (Figure 1b). This scanning mechanism will be extensively described in the next sections. A specific immunocomplex formed between a large and a small particle, due to the presence of even a single $\mathrm{Ag}$ on the large particle, binds the Ag-carrying large particle to the substrate (Figure 1c). A counting of the number of captured large particles provides a direct measure of the Agconcentration in the sample (Figure 1d).

Chemicals and materials. 4-inch $550 \mu \mathrm{m}$ thick Si and float glass wafers, de-ionized water (DIW) were obtained from the Center of Micro- and Nanotechnology of EPFL. GM 1075 SU-8 negative photoresist was purchased from Gersteltec (Pully, Switzerland). AZ ECI 3027 positive photoresist was purchased from AZ Electronic Materials (Wiesbaden, Germany). PDMS Sylgard 184 was 
acquired from Dow Corning (Wiesbaden, Germany). $1 \mathrm{~mL}$ borosilicate H-TLL-PE syringes were purchased from Innovative Labor Systeme (Stutzerbach, Germany). Micro-line ethyl vinyl acetate tube with $0.51 \mathrm{~mm}$ inner and $1.52 \mathrm{~mm}$ outside diameters was bought from Fisher Scientific (Wohlen, Switzerland). Neodymium magnets were acquired from Webcraft (Uster, Switzerland): (i) disc magnets with $6 \mathrm{~mm}$ diameter, $3 \mathrm{~mm}$ thickness (product no: S-06-03-N, magnet-magnet attraction force: $\sim 62 \mathrm{~N}$ ); (ii) disc magnets with $10 \mathrm{~mm}$ diameter, $3 \mathrm{~mm}$ thickness (product no: $\mathrm{S}$ 10-03-N, magnet-magnet attraction force: $\sim 18 \mathrm{~N}$ ); (iii) ring magnets with $4 \mathrm{~mm}$ inner and $10 \mathrm{~mm}$ outer diameters, $5 \mathrm{~mm}$ thickness (product no: R-10-04-05-N, magnet-magnet attraction force: $25 \mathrm{~N}$ ). The APTES solution (product no. 440140), Phosphate Buffered Saline (PBS) 10x concentrate solution (product no. 5493), Tween-20 (product no. 1379) and biotin (product no. B4501) were purchased from Sigma-Aldrich (Buchs, Switzerland). Dynabeads MyOne Streptavidin C1 (small particles), Dynabeads M-280 Streptavidin (large particles), recombinant mouse TNF- $\alpha$, biotinylated polyclonal rabbit Ab specific to mouse TNF- $\alpha$ and FBS dialyzed were purchased from Life Technologies (Zug, Switzerland). Biotinylated goat anti-streptavidin and streptavidin were obtained from Vector Laboratories (Reactolab SA, Servion, Switzerland). Fetal Bovine Serum (FBS) standard quality was bought from PAA Laboratories (Chemie Brunschwig AG, Basel, Switzerland) and Pluronic F-127 was purchased from BASF (Basel, Switzerland). A PBS (0.15 M NaCl, $0.01 \mathrm{M}$ phosphate, $\mathrm{pH}$ 7.4) solution was prepared by diluting a PBS 10x concentrate solution. PBS-Tween $1 \%$ solution was made by diluting $1 \%$ (volume / volume) Tween-20 in PBS. A buffer solution was prepared by diluting $0.08 \%$ (weight/volume) Pluronic F127 in PBS. To reach Ag concentration levels used in the experiments, the biotinylated antistreptavidin was diluted in PBS or FBS dialyzed and mouse TNF- $\alpha$ was prepared in FBS standard quality. 
Fabrication of the glass detection chip via electrostatic self-assembly of magnetic particles. $0.6 \mu \mathrm{m}$ thick AZ ECI 1512 photoresist was patterned on a glass wafer using standard photolithography techniques. ${ }^{31}$ The pattern features a $250 \mu \mathrm{m} \times 2.5 \mathrm{~mm}$ area containing $\sim 8000$ dots, $1 \mu \mathrm{m}$ in diameter, with a $10 \mu \mathrm{m}$ inter-distance between adjacent dots. The wafer was diced into chips with $2.8 \mathrm{~cm} \times 2.5 \mathrm{~cm}$ area. The chip surface was activated in air plasma for $40 \mathrm{~s}$ at 12 W and 1 mbar pressure. $1 \%$ (volume / volume) APTES solution diluted in DIW was spin-coated at $5000 \mathrm{rpm}$. The chip was baked at $100^{\circ} \mathrm{C}$ for $10 \mathrm{~min}$. The resist was ultrasonically removed in acetone for $2 \mathrm{~min}$. The chip having a pattern of $1.5 \mu \mathrm{m}$ APTES dots was stored at $-18^{\circ} \mathrm{C}$ until further use. For small particle patterning, the chip was reversibly bonded to a dedicated PDMS chip, containing a microfluidic channel with $60 \mu \mathrm{m}$ height and $500 \mu \mathrm{m}$ width. The glass detection chip and this PDMS chip were tightly clamped for leak-proof sealing. For electrostatic binding of the small particles to the APTES dot pattern, a $10 \mu \mathrm{L}$ solution of small particles was introduced inside the microfluidic channel at a $150 \mathrm{~nL} \mathrm{~s}^{-1}$ flow rate using a syringe pump. Hereafter, a $30 \mu \mathrm{L}$ PBST $1 \%$ washing step was conducted at $5 \mu \mathrm{L} \mathrm{s}^{-1}$. Finally, the glass detection chip was separated from the PDMS and was ready to be clamped to the PDMS microfluidic chip.

Fabrication of the microfluidic chip for the experimental analysis of the magnetic-particle scanning. PDMS microchannels were replicated using SU-8 microstructures on Si wafers. First a $60 \mu \mathrm{m}$-thick SU-8 layer was patterned through photolithography processes. Liquid PDMS mixture (10:1 base:cross-linker weight ratio) was degassed, poured on the SU- 8 mold and cured at $100{ }^{\circ} \mathrm{C}$ for $1 \mathrm{~h}$. The cured PDMS layer was peeled off from the mold and the fluid injection holes were punched through the chip. The PDMS chip was then surface-activated with air plasma at $12 \mathrm{~W}$ for 1 min, together with a glass support, to which the PDMS was successively bonded. For experiments with magnetic particles patterned on APTES (i.e. glass detection chips), the cured 
PDMS piece was instead clamped to the glass substrate by using a custom-made PMMA holder. PDMS channels used for these analyses size $60 \mu \mathrm{m}$ in height and $250 \mu \mathrm{m}$ in width.

\section{Magneto-microfluidic setup for the experimental analysis of the magnetic-particle scanning.}

$1 \mu \mathrm{m}$-diameter superparamagnetic particles were randomly patterned at the bottom of the microfluidic channel by strong unspecific adsorption on the glass substrate. A ring magnet (10 mm outer diameter, $4 \mathrm{~mm}$ inner diameter, $5 \mathrm{~mm}$ height) was positioned below the microfluidic chip, at $10 \mathrm{~mm}$ distance from the channel bottom, by using a custom-built PMMA holder. This resulted in a constant vertical field induction $B_{\text {ext }, \mathrm{z}}=30 \mathrm{mT}$ over the substrate detection zone at the level of the immobilized particles, as measured via Gauss meter and simulated by Finite Element Method (FEM). The full assembly was flipped upside down and positioned on the stage of an inverted microscope (Zeiss Axiovert S100), to allow light transmission through the ring magnet and light collection from below the chip, via a high magnification objective (LD EC Epiplan-Neofluar, 50x, Zeiss). The flow manipulation in the microfluidic channel was performed by precision syringe pumps (Nemesys, Cetoni, Korbussen, Germany). $3 \mu \mathrm{m}$-diameter superparamagnetic particles were diluted in buffer solution to a final concentration of $0.01 \mathrm{mg}\left(\sim 7 \times 10^{4}\right)$ particles per $\mathrm{mL}$. The particle suspension was then injected in the microchannel at $100 \mathrm{~nL} \mathrm{~s}^{-1}$ flow rate. High-speed camera tracking (MC-1363 camera, Mikrotron, Unterschleissheim, Germany) was used to accurately monitor individual particles during their transport in the microchannel, at 1200 framesper-second rate. The gradient of the external magnetic field in the direction normal to the substrate plane resulted in the effective magnetophoretic attraction of the particles towards the glass substrate. The field intensity was chosen to be weak enough not to capture large particles on the glass surface, where they could hence still move and being transported by the flow across the small 
particle pattern. Image analyses were performed using the ImageJ software (Bethesda, Maryland, USA).

\section{RESULTS AND DISCUSSION}

Magnetic particle-scanning model. In this section, we describe the physics of the interparticle interactions leading to the particle-scanning mechanism. When two superparamagnetic particles are placed in a constant magnetic induction $\mathbf{B}$, they acquire each a magnetic moment, $\mathbf{m}_{\mathbf{1}}(\mathbf{B})$ and $\mathbf{m}_{2}$ (B) respectively, and interact via dipolar forces. If the first particle is immobilized on a substrate that is normal to $\mathbf{B}$, the second particle will be subjected to a torque, the size of which can be calculated as the first angular derivative of the dipolar interaction energy between the two particles, as follows:

$$
\tau_{\text {mag,dip }}=-\frac{\mathrm{d}}{\mathrm{d} \theta}\left[\frac{\mu_{0}}{4 \pi\left\|\mathbf{r}_{\mathbf{1}, \mathbf{2}}\right\|^{3}}\left(\mathbf{m}_{\mathbf{1}} \cdot \mathbf{m}_{\mathbf{2}}-\frac{3}{\left\|\mathbf{r}_{\mathbf{1}, 2}\right\|^{2}}\left(\mathbf{r}_{\mathbf{1}, \mathbf{2}} \cdot \mathbf{m}_{\mathbf{1}}\right)\left(\mathbf{r}_{\mathbf{1}, \mathbf{2}} \cdot \mathbf{m}_{\mathbf{2}}\right)\right)\right]
$$

where $\theta$ is the angle formed by the position vector between the center of mass of the two particles $\left(\mathbf{r}_{1,2}\right)$ and the substrate plane (see Figure $2 \mathrm{a}$ ), $\mu_{0}=4 \pi \times 10^{-7} \mathrm{~T} \mathrm{~m} \mathrm{~A}^{-1}$ is the permeability of vacuum, and $\mathbf{m}_{1}$ and $\mathbf{m}_{2}$ are the magnetic moments of the two particles that we consider to be point-like dipoles. A stable situation is reached when $\mathbf{m}_{\mathbf{1}}$ and $\mathbf{m}_{\mathbf{2}}$ are aligned along the field direction $(\theta=$ $90^{\circ}$ ). Figure $2 \mathrm{~b}$ shows the intensity of the magnetic induction $\mathbf{B}$ and the spatial distribution of the magnetic induction lines within a large and small magnetic particle for an externally applied induction of $30 \mathrm{mT}$, as calculated using a 3D Finite Element Method (FEM) simulation (Comsol Multiphysics). We used a magnetic susceptibility $\chi$ of 0.7 and 1.4 for the large and small magnetic particle, respectively. ${ }^{32}$ Our calculation shows that the perturbation of the vertical magnetic field 
lines due to the presence of the particles is minimum, thus supporting the assumption that both magnetic moments are oriented along the z-direction, as implicitly assumed in Equation (1).

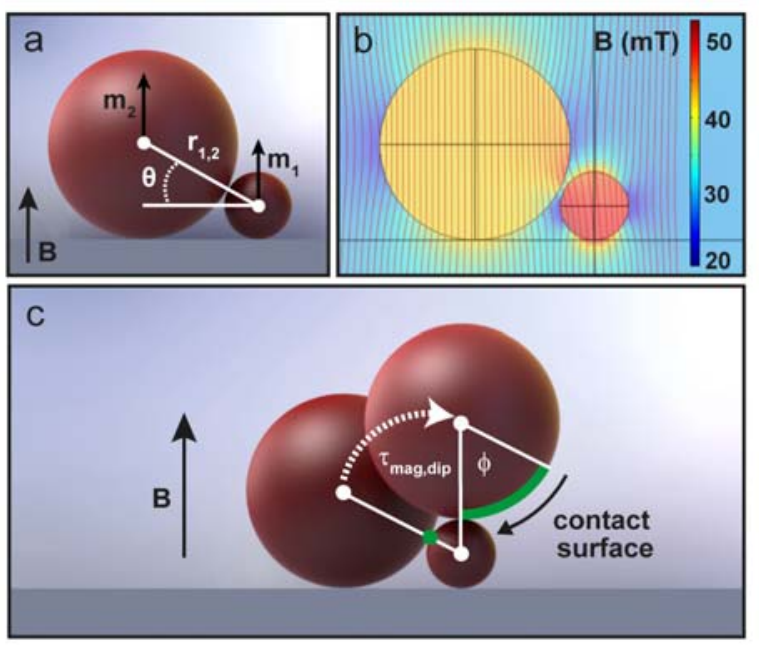

Figure 2. (a) Schematic of a small and a large magnetic particle with magnetic moment $\mathbf{m}_{\mathbf{1}}$ and $\mathbf{m}_{\mathbf{2}}$, respectively, and indicating the angle $\theta$ and the position vector $\mathbf{r}_{\mathbf{1 , 2}}$. (b) FEM simulation of the field lines for a large and small magnetic particle, placed in an external magnetic induction of 30 mT. (c) Contact of the large particle sliding over a small particle under influence of the torque $\tau_{\text {mag,dip }}$, as indicated by the arc corresponding to the angle $\delta$.

As domain reorientations in a magnetic particle, which consists of an ensemble of magnetic nanocrystals in a non-magnetic matrix, are relatively $\operatorname{slow}^{33}, \mathbf{m}_{\mathbf{1}}$ and $\mathbf{m}_{\mathbf{2}}$ are not expected to change their orientation with respect to the particle surfaces on the time scale of their interaction, leading to a "pinning" of the magnetic moments to the particles. As a consequence, an increasing part of the large particle will be in contact with the small particle, when the former slides over the latter under influence of the torque $\tau_{\text {mag,dip }}$. The distance on the large particle surface over which this contact takes place is indicated by the arc defined by the angle $\delta$ (see Figure 2c). 
Moreover, as the induced displacement of the large particle occurs in a liquid with viscosity $\eta$, a “magnetic tangential velocity" $v_{m a g, t a n}$ arises from instantaneous equilibrium between magnetic and drag forces applied to the particle, as follows:

$$
v_{\text {mag,tan }}=-\frac{\tau_{m a g, d i p}}{\left\|\mathbf{r}_{\mathbf{1}, 2}\right\| \cdot 6 \pi \eta R f_{D}}
$$

where $R$ is the particle radius and $f_{D}$ is the corrective drag coefficient taking into account the vicinity of the particles to the substrate. ${ }^{19}$ When the substrate is placed at the bottom of a microfluidic channel, in which a constant flow is applied, a Poiseuille flow develops in the microchannel and the local fluid velocity $\mathbf{v}$ can be calculated by solving the Navier-Stokes equation for incompressible flow in the microfluidic channel. ${ }^{34}$ An additional viscous drag force will act therefore on the moving particle, the size of which is calculated as:

$$
F_{\text {drag }}=6 \pi \eta R\|\mathbf{v}\| f_{D}
$$

with $\mathbf{v}$ conveniently taken as the velocity of the unperturbed Poiseuille flow at the center of mass of the particle. ${ }^{19}$ Because of the parabolic velocity profile in the microchannel, the drag force acting on the large particle will be strongest once it is on top of the small particle. This microfluidic viscous force can be tuned to detach the large particle, when no additional tethering forces are introduced to keep the two particles linked. ${ }^{35}$

Experimental observation of the inter-particle interaction. To verify our theoretical model experimentally, we have randomly patterned small particles on a glass substrate and placed it on our magneto-microfluidic platform. A detailed description of the experimental method is reported in SI. The presence of the permanent magnet resulted in a constant vertical field induction $B_{\text {ext }, z}=$ $30 \mathrm{mT}$ at the level of the immobilized particles, as measured via a Gauss meter and simulated by 
FEM (see Figure S1). A diluted suspension of large superparamagnetic particles is injected in the microchannel at $100 \mathrm{~nL} \mathrm{~s}^{-1}$ flow rate. Under these magnetic and drag force conditions, large particles gently slide over the substrate and interact with the small particles, as described in the previous section and shown in Video (.AVI format) of the Supporting Information (SI).
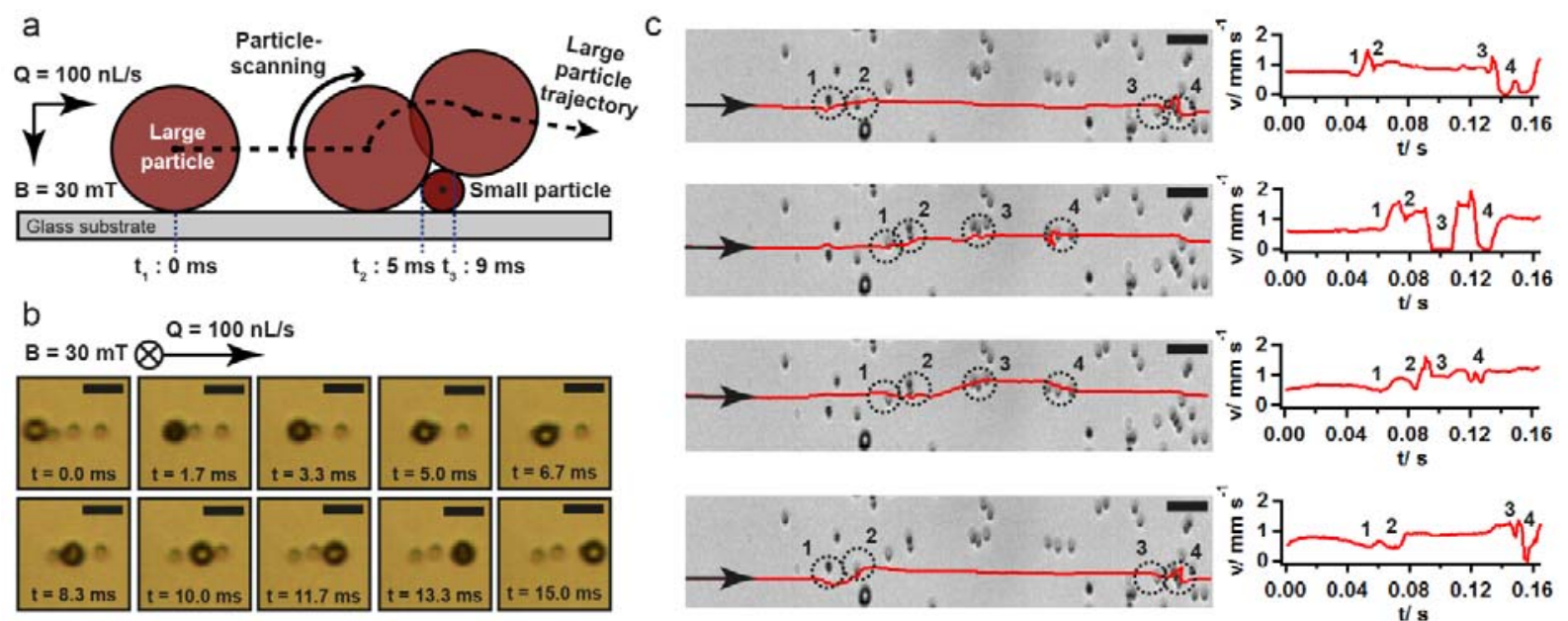

Figure 3. (a) Schematic motion of a large particle in the detection area in the presence of a small particle, as obtained from a Matlab simulation, for an external magnetic field $\mathrm{B}=30 \mathrm{mT}$ and flow rate $\mathrm{Q}=100 \mathrm{~nL} \mathrm{~s}^{-1}$. A total contact time of $\sim 4 \mathrm{~ms}$ is estimated between the two particles. (b) Motion of a large particle over the detection area, for $\mathrm{B}=30 \mathrm{mT}, \mathrm{Q}=100 \mathrm{~nL} \mathrm{~s}^{-1}$, as experimentally observed via a high-speed camera. Scale bar $=5 \mu \mathrm{m}$. (c) Experimental tracks and velocity profiles for four different large particles, for $\mathrm{B}=30 \mathrm{mT}, \mathrm{Q}=100 \mathrm{~nL} \mathrm{~s}^{-1}$, taken from the video shown in the SI. At each encounter with an immobilized small particle, a large particle undergoes both in-plane and out-of plane displacements. Scale bar $=10 \mu \mathrm{m}$. 
Figure $3 \mathrm{a}$ describes the behavior of a large particle in vicinity of a small particle, as obtained from the modeling corresponding to the described experimental conditions. Three distinct phases are defined for an inter-particle contact event:

i. transport of a large particle by viscous drag forces (in the time interval $t_{2}-t_{1}$ );

ii. encounter with a small particle (at $\mathrm{t}_{2}$ ) and contact during a time interval $t_{3}-t_{2}$, when the large particle is subjected to the magnetic torque; and

iii. large particle release inside the channel, in case no tethering force is linking the two particles.

The contact time between the two particles is estimated by taking into account two contributions: the local velocity $\mathbf{v}$ of the fluid, as simulated by FEM, and the magnetic tangential velocity $v_{\text {mag, } t a n}$ of the rotatory movement, calculated in Matlab using (2). Neither in the experiment nor in the model, ligand-receptor forces between the two particles are included, yet. A contact time of approximately $4 \mathrm{~ms}$ between the two particle surfaces is calculated, in good agreement with the experimental result of Figure $3 b$.

In Figure 3c we report the trajectories of four different large particles, taken from the video shown in the SI, as they flow over the small particle-patterned area. Multiple in-plane reorientations and trajectory deviations of each flowing particle can be observed at each encounter with a small particle. Moreover, the velocity plot associated to each trajectory allows extracting further information about the out-of-plane particle movements. Large particles flow at relatively constant velocity $\left(0.8-1 \mathrm{~mm} \mathrm{~s}^{-1}\right)$ on the glass surface and abruptly decelerate while in close proximity to any immobilized particle. The small peaks in the velocity profiles correspond to sudden increases 
of the velocity upon detachment of a large particle sitting on top of a small particle (time $t_{3}$ in Figure 3a), where hydrodynamic flow and drag force is maximum.
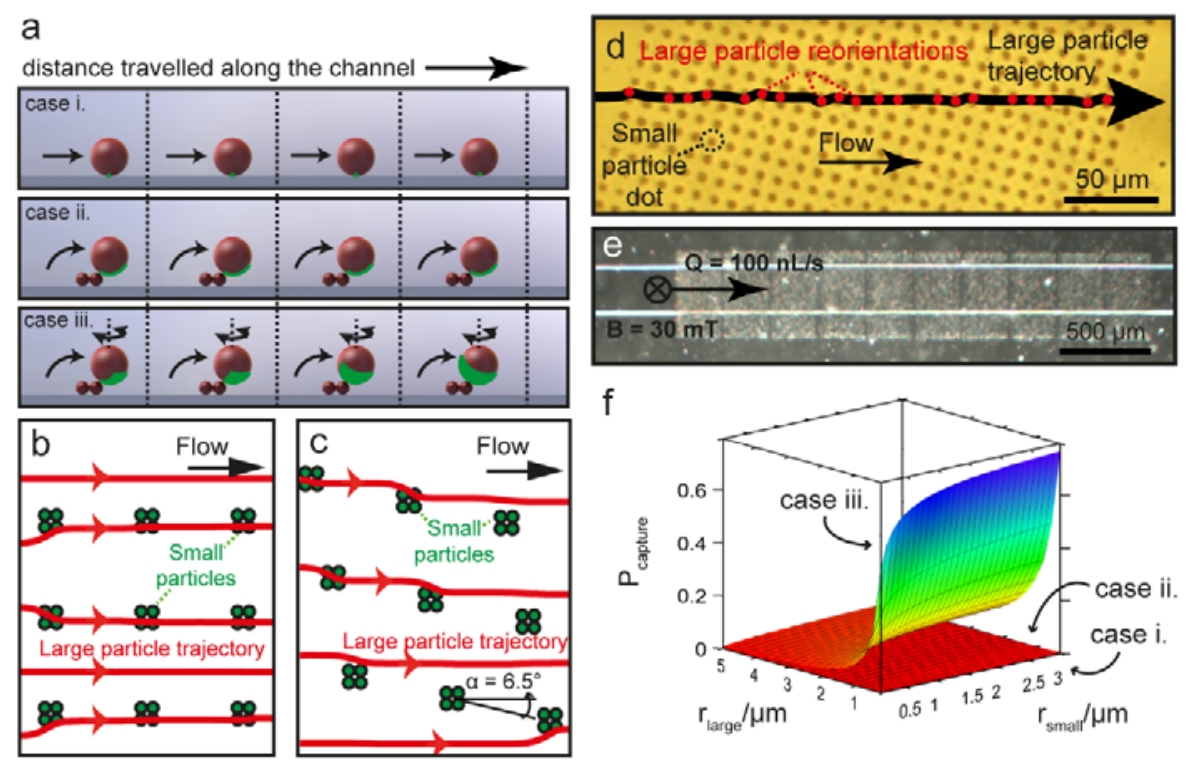

Figure 4. (a) Large magnetic particle moving in a magnetic field that pins its magnetic moment (top) in absence of immobilized small particles, (center) in presence of small particles, but only moving in the symmetry plane (no lateral displacements) and (bottom) under our experimental conditions, where lateral displacements of the large particle occur as well. In the last case, a different section of the large particle surface is explored at each interaction with a small particle dot. (b,c) Simulated large particle trajectories on (b) a non-tilted and (c) a $6.5^{\circ}$ tilted regular array of 4 small particles per pattern, iteratively traced by combining FEM simulations and Matlab analytical calculations (see SI). The tilted pattern guarantees more frequent encounters between immobilized small particles and flowing large particles. (d) Trajectory of a large particle on a tilted small particle array, under our standard experimental conditions ( $\left.B_{\text {ext }, z}=27-30 \mathrm{mT}, Q=100 \mathrm{~nL} \mathrm{~s}^{-1}\right)$, as experimentally observed using a high-speed camera. Red dots are used to visualize the large particle reorientation events ( $\sim 20$ over a $250 \mu \mathrm{m}$-long array). (e) Optical microscopy photograph 
of the particle array used for protein detection experiments. (f) $\mathrm{P}_{\text {capture }}$ calculated for the three situations of Figure $4 \mathrm{a}$ as function of the radii of the large and small particle using Equation (S1),(S2) and (S3), taking $\mathrm{N}=1$ and $\mathrm{r}_{\text {ligand }}=2.2 \mathrm{~nm}$. The cases i. and ii. are not easily distinguishable on this graph (even though case i. always induces a smaller $\mathrm{P}_{\text {capture }}$ than case ii. by at least two orders of magnitude) so that this representation merely emphasizes the increased $\mathrm{P}_{\text {capture }}$ from case iii.

Increasing the ligand-receptor encounter probability by particle scanning. In this section we estimate the gain in ligand-receptor encounter probability induced by the scanning mechanism, under our standard experimental conditions. Figure $2 \mathrm{c}$ already showed the increase of contact area of a large particle with an immobilized small particle due to the sliding motion induced by the magnetic torque. In this schematic picture all the elements of the system were aligned along a symmetry plane defined by the flow direction and the vertical axis, i.e. the trajectory of the large particle is constrained within this plane. However, in reality, as already suggested by Figure 3c, this symmetry is rarely conserved. The contact between a large and a small particle mainly occurs out of this symmetry plane, giving rise to a lateral displacement and extra lateral components of the magnetic and hydrodynamic forces. In successive contact events with a number of small particles, this gives rise to the scanning of even a larger part of the surface of the large particle than shown in Figure 2c. Figure 4a, case i. (top) shows the contact of the large particle while moving in the presence of the magnetic field that pins the magnetic moment, but without the immobilized small particles. In this case, the particle simply slides over the flat substrate and "probe" it with only one point, because of the spherical geometry, as already demonstrated by others. ${ }^{36}$ Therefore the ligand-receptor encounter probability is expected to be low, especially for single molecule detection. When the large particle moves in the symmetry plane and small particles 
are present, the contact point describes a line on the surface of the large particle (Figure 2c), increasing somewhat the chance for ligand-receptor encounter (Figure 4a, case ii. (center)). Even when multiple small particles are explored, as the orientation of the large particle is not altered, the same portion of the large particle surface is each time scanned. If we now consider the real case, where lateral displacements of the large particle occur, a different section of the large particle surface is explored at each interaction with a small particle dot (Figure 4a, case iii. (bottom)). It is interesting to note that up to now we assumed that the particles are solely exposed to magnetic and hydrodynamic forces. Nevertheless, other surface interactions of different origin, such as electrostatic, chemical or friction forces, may introduce an additional torque ${ }^{37}$, which would even be more beneficial for enhancing this reorientation phenomenon.

The attempt frequency of ligand-receptor binding corresponds to the rate of large particle reorientation events and can be strongly enhanced by precisely controlling the arrangement of the immobilized small particles. In particular, we designed different regular patterns of immobilized small particles and developed a particle-tracking Matlab code to predict the trajectory of the large particles over them (see SI). Figure $4 \mathrm{~b}$ shows an array of isolated dots of four small particles at 9 $\mu \mathrm{m}$ inter-distance, allowing a high density of small particles while still avoiding magnetic crossinteraction between adjacent dots, with the trajectories of five large particles moving over the pattern. A $6.5^{\circ}$ tilting angle of the dot pattern with respect to the flow direction (Figure 4c) was found to be a good choice for increasing the number of encounters, in comparison to the geometry shown in Figure 4b. We fabricated this optimized small particle array via electrostatic selfassembly on a micropatterned aminopropyl-trietoxysilane (APTES) layer. ${ }^{38}$ The full array is made up of ten $250 \mu \mathrm{m}$ x $250 \mu \mathrm{m}$ sections, for a total number of $\sim 8000$ small particle dots. Under our standard experimental conditions $\left(B_{e x t, z}=27-30 \mathrm{mT}, Q=100 \mathrm{~nL} \mathrm{~s}^{-1}\right)$, we found that a single large 
particle encounters on average 20 small particle dots over the length of each section (Figure 4d), resulting in about 200 encounters over the full length of the array (Figure 4e).

To estimate the gain in ligand-receptor encounter probability induced by the scanning mechanism, we calculated the probability to successfully bind the large particle $P_{\text {capture }}$ for the three cases described in Figure 4a, adapted to our real experimental conditions. A detailed description of this estimation is reported in SI. As shown in Figure 4f, the scanning mechanism is expected to introduce a dramatic enhancement of the particle capture probability.

While this simple model already shows that both the scanning and the reorientation of the large particle improve the capture probability, however, this is only an approximation for the behavior of the real system. Indeed, in this estimation it was assumed that the changes in the orientation of the particle between the interactions with the immobilized surfaces were completely random. In our system, these interactions are more appropriately described by a single point of contact that is randomly diffusing over the surface of the particle until it hits a bound Ag. A random walk description would therefore be a much better approximation than a succession of independent tests of the stochastic variable. Additionally, the orientation of the particle between these events is primarily controlled by the local magnetic field and the magnetic moment of the large particle. This phenomenon is likely to prevent the full surface of the particle from being scanned by the immobilized structure (see SI), as the moment of the particle is pinned along the direction of the magnetic field after each encounter with a small particle dot (but the particle can still rotate around the axis defined by its magnetic moment, thus increasing the degrees of freedom of the model). Hence, this analysis suggests that the interactions of the particle with the immobilized magnetic structures can be modeled as a random walk over a fraction of the particle surface, centered on its magnetic moment. This is however only an approximation of this phenomenon. Indeed, during a 
true random walk, the probability for the next movement is the same for any direction. In our case, as the molecule is moving in the direction of the flow, a movement of the point of contact on the surface of the particle in the direction of the flow is more likely. Nevertheless, as detailed in the previous description, the system is not perfectly symmetric, and random rotation along the $\mathbf{z}$ axis, changes in direction, out-of-plane contacts, etc. are all expected to enhance the reorientation rate of the bead, so that the trajectory of the point of contact on the surface of the sphere is not a straight line in the direction of the flow, but is actually randomized by the constant rotation and reorientation of the particle. Hence, the point of contact could potentially then move in any direction, even though the direction of the flow would be the one associated to the highest probability. We assumed that this behaviour could be modelled by a random-walk. Overall, even though the random-walk is still an approximation of the actual phenomenon, it was found to describe accurately the behavior of the system and to be in good agreement with the experimental data, thus supporting this hypothesis (vide infra, Figure 7). More precisely, this particle scanning mechanism contributes at explaining the very low limit of detection and wide dynamic range of the doseresponse curve of a previously reported surface coverage assay. ${ }^{18}$

Evaluation of the particle binding along the detection zone. As the number of particles specifically captured on each section of the small particle dots landscape (see Figure 1d) can be easily assessed by microscopic observation, this analysis allows for the study of the capture probability as a function of the distance travelled within the channel. As shown in the insert of Figure 5a, two different regimes can be identified when a particle is injected into the detection channel. First, it is transported by the parabolic flow, while magnetophoretically attracted to the bottom of the channel by the external magnetic field. We refer to this as the 'falling' regime. The graph of Figure 5a shows the fraction of the large particle population that has reached the substrate 
as a function of the distance in the microchannel for our typical experimental conditions (see SI for details of the model calculation). Once a particle has 'landed' on the substrate, the scanning mechanism starts, during which the former slides over the surface of the substrate, at a speed $v_{0} \sim 0.8-1 \mathrm{~mm} \mathrm{~s}^{-1}$ for our typical experimental conditions, until it is eventually captured by a small particle dot.

Figure $5 \mathrm{~b}$ shows the experimental fraction of large particles captured as a function of the channel distance, for different concentrations of TNF- $\alpha$ molecules spiked in fetal bovine serum (FBS). To emphasize the capability of the device for detection of very low amounts of target molecules, we report, instead of the concentration, the number of molecules present in the $5 \mu \mathrm{L}$ sample injected in the chip $N_{A g}$. The fraction of large particles is experimentally obtained by simply counting the number of particles present on each $250 \mu \mathrm{m}$ long section of the small particle dot pattern (see Figure 4e). As expected, this fraction increases linearly with distance, and the slope of this line increases with $N_{A g}$. In our experiments, the captured fraction $v s$. distance curves show an initial offset at the beginning of the sensing region (i.e. a non-zero fraction at $x=0 \mathrm{~mm}$ on Figure $5 \mathrm{~b}$ ), increasing with $N_{A g}$. This offset can be explained by the important number of large particles that have landed in the micro-channel region upstream of the small particle landscape and are moving over the substrate. Upon their encounter with the first small particle detection section, a relatively high amount of large particles is therefore initially captured (see SI for details).

The curves of Figure $5 \mathrm{~b}$ are linearly fitted and the slope $\left(\equiv \operatorname{slope}\left(N_{A g}\right)\right)$ of these fits is presented as a function of $N_{A g}$ in Figure 5c. Very strikingly, the dependence of slope $\left(N_{A g}\right)$ on $N_{A g}$ is strongly sub-linear, i.e. lower concentrations are much more effectively detected than higher ones. We will see in the next section that the physical quantity slope $\left(N_{A g}\right)$ can be conveniently used to quantify $P_{\text {capture }}$ Furthermore, the result output $\left(N_{A g}\right)$ of our assay, defined as the fraction of all injected large 
particles captured over the ten small particle detection sections, is found in good approximation to be proportional to slope $\left(N_{A g}\right)$. The coefficient of proportionality was evaluated as $4.4 \pm 0.5 \mathrm{~mm}$ (for the 5 datasets corresponding to concentrations above the LOD, average \pm standard deviation (SD)). The variation of this coefficient is found to be very small (about $11 \%$ of the average) over the 8 orders of magnitude considered in our assay.
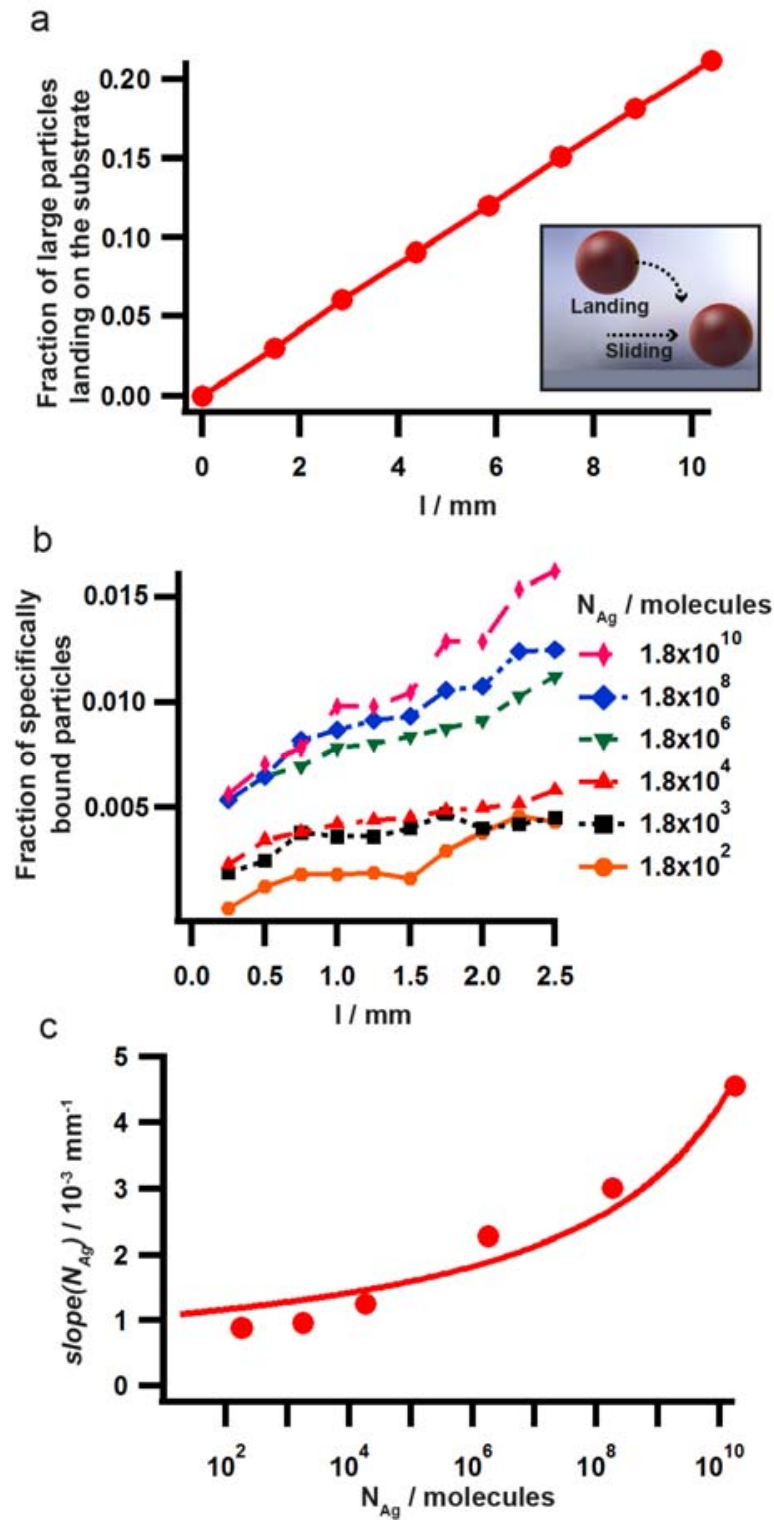
Figure 5. Study of the capture efficiency vs. length of the channel. (a) Calculation of the fraction of the large particles that have landed on the substrate as a function of the distance in the microchannel for our standard experimental conditions. Insert: schematic illustration of the 'falling' and 'sliding' regimes of a large particle. (b) Experimental fraction of large particles captured as a function of the channel distance, for different concentrations of TNF- $\alpha$ molecules spiked in fetal bovine serum (FBS) under our standard experimental conditions. (c) Slopes obtained from the experimental curves shown in (b) as a function of the number of Ag molecules. The full line shows a fit of the experimental data with the model established in Equation (4).

Random walk description of the scanning process. From the previous analysis, the interactions between the large particle and the immobilized small particle dots can be approximated by a random walk of the contact point over the surface of the large particle, as illustrated in Figure 6a. This stochastic displacement is assumed to stop when the point of contact between a large and a small particle coincides with the position of an Ag bound to the large particle. Mathematically, the evolution of the bead trajectory can be described, on average, by the mean first passage time of a point randomly walking over the surface of a sphere, as shown in Figure 6a. If we also assume that the amount of Ag bound to the surface of a sphere is described by a Langmuir equation, the amount of large beads captured over the surface of the device for a given $N_{A g}$, $\equiv \operatorname{output}\left(N_{A g}\right)$, can be shown to have the following form (see detailed calculations in SI):

$$
\operatorname{output}\left(N_{A g}\right)=\frac{\beta}{\ln \left(\gamma \frac{\alpha N_{A g}}{1+\alpha N_{A g}}\right)}
$$

$\operatorname{output}\left(N_{A g}\right)$ is here the final detection signal of the device (i.e. the number of captured beads counted by the operator at the end of the experiment) and is linked through this relationship to the 
input of the detection device $N_{a g}$. Hence, Equation 4 is expected to totally describe the behavior of the device. The variables $\alpha, \beta$ and $\gamma$ are constants accounting for different phenomena:

- $\alpha$ is the Langmuir constant, characterizing the affinity of the Ag for its receptor. For instance, $1 / \alpha$ is the amount of Ags required to cover half of the sphere with bound Ags. Here it is also expected to partly describe the efficacy of the mixing;

- $\beta$ is a negative constant depending on the characteristic of the system. From Equation (S17), it decreases with the flow velocity and increases with the binding probability. From Equation (S11), it also depends on the factor B, with characterizes the mean first passage time;

- $\quad \gamma$ can be obtained from Equation (S12), and characterizes the distance between the bound Ags, for an even distribution over the surface of the sphere. It depends largely on the geometry of the large beads.

Interestingly, Equation (4) is similar to the one obtained for the binding time of a single Agcarrying particle, which is allowed to rotationally diffuse when in contact with another particle fully covered with receptors. ${ }^{39}$ As expected output $\left(N_{A g}=0\right)=0$. However, it diverges when $\gamma \frac{\alpha N_{A g}}{1+\alpha N_{A g}} \rightarrow 1$, as, in this condition, the low binding hypothesis allowing the linearization of Equation (S10) is not satisfied anymore.

The experimental values of slope $\left(N_{A g}\right)$ were fit with Equation (4), as shown in Figure $5 \mathrm{c}(\alpha=$ $3.9 \times 10^{-13}$ molecules $\left.^{-1}, \beta=-2.9 \times 10^{-2} \mathrm{~mm}^{-1}, \gamma=2.7 \times 10^{-1}\right)$. The Langmuir adsorption constant $\alpha$ normally describes the binding affinity of the substrate to its receptor, but here also accounts for steric hindrance effects on the large particle surface. Based on the values obtained from our fitting, the variations of the Ag coverage on the large particles were computed using the Langmuir 
equation presented in Equation (S14) and are shown on Figure 6b. It shows that the surface of a large particle would be saturated (i.e. coverage $>90 \%$ ) when $>10^{13} \mathrm{Ag}$ are injected, corresponding to $\sim 6 \times 10^{9} \mathrm{Ag}$ per particle. Interestingly, we have calculated that, assuming a radius of $2.2 \mathrm{~nm}$ for a single TNF- $\alpha$ molecule ${ }^{28}, 8 \times 10^{5}$ TNF- $\alpha$ molecules only are required to cover a $2.8 \mu$ m diameter particle. This indicates that the Ag capture on a large particle surface for the very large concentrations necessarily will be less efficient.
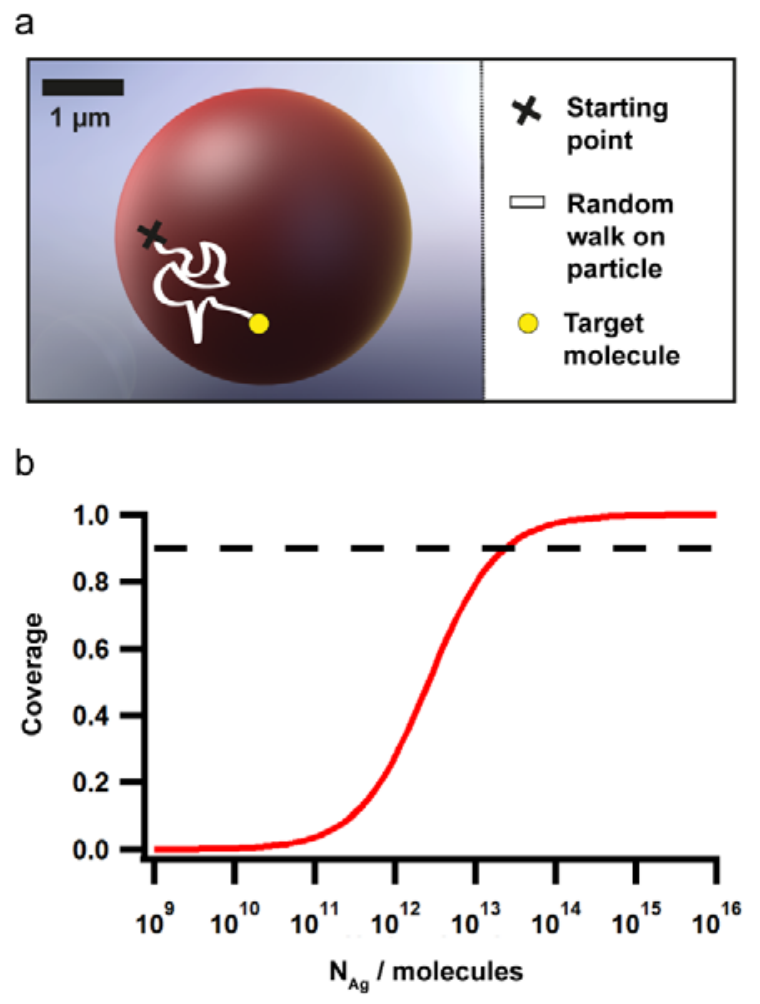

Figure 6. (a) Displacements of the large particle over a substrate with immobilized small particle dots, approximated by a random walk of the contact point over the surface of the large particle, corresponding to the situation of Figure $4 \mathrm{a}$ (bottom). (b) Calculation of the coverage fraction of the Ag over a large particle surface, assuming this mechanism follows a Langmuir isotherm with $\alpha=$ 
$3.9 \times 10^{-13}$ molecules $^{-1}$. The horizontal dotted line corresponds to $90 \%$ coverage obtained from the Langmuir adsorption model, under our standard experimental conditions.

Explanation of protein detection experiments. The experimental dose-response curves (data of ref. $\left.{ }^{18}\right)$ are now analyzed using our random walk model, in which output $\left(N_{A g}\right)$ was expressed by Equation (4) under the assumption that the $1^{\text {st }}$ order kinetics of the reaction can be approximated with a linear behavior (see SI).

The biotin/streptavidin binding experiments performed in FBS are fit with Equation (4), as shown on Figure 7a. Furthermore, the data obtained for the detection of TNF- $\alpha$ in FBS, which was presented and studied in a previous paper ${ }^{18}$, are also fit with this model (Figure $7 \mathrm{~b}$ ). The different values obtained from the fitting, i.e. the parameters $\alpha, \beta$ and $\gamma$ and the maximum coverage of the particles over the range of concentrations considered in the experiment, as described by the Langmuir Equation, are presented in Table 1. 

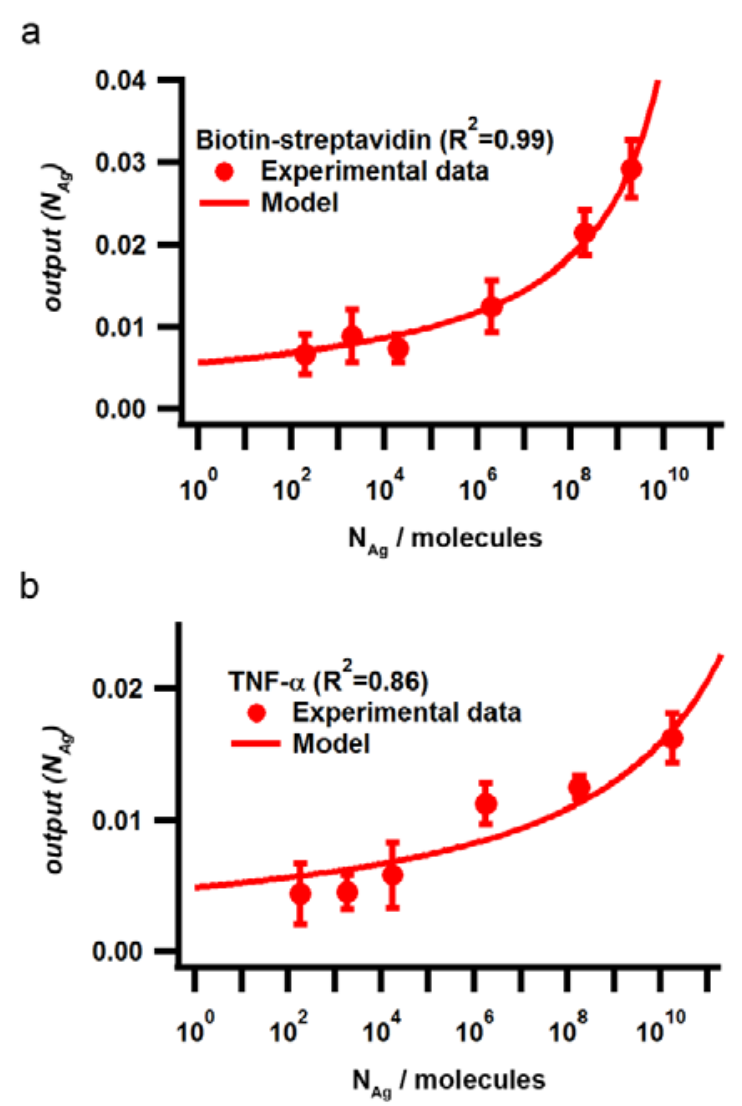

Figure 7. Experimental data for (a) biotinylated anti-streptavidin and (b) TNF- $\alpha$ in FBS (n=3-4, the error bars are SD). The solid lines are fit to the experimental values following the expression for output $\left(N_{A g}\right)$ detailed above in Equation (12). The $\mathrm{R}^{2}$ values are 0.99 and 0.86 for the fittings of the biotin/streptavidin and TNF- $\alpha$ datasets, respectively.

The critical factor for this fitting, the Langmuir adsorption constant $\alpha$, is found to be much higher for the biotin/streptavidin ligand/receptor system than the one computed above for the TNF- $\alpha$ system (see Table 1). This result is in good agreement with the very high binding affinity of biotin to streptavidin, even in comparison to an $\mathrm{Ag} / \mathrm{Ab}$ system. Table 1 shows, in addition to the fit parameters, some representative dissociation constants $K_{D}$ reported in the literature for the different systems detailed in this study. As mentioned above, $\alpha$ is an indicator of the efficacy of 
the Ag binding. A high $\alpha$ and a low $K_{D}$ indicate a high binding affinity, as observed from the values shown on Table 1. Furthermore, the low values found for $\alpha$ are still in good agreement with the central assumption in the random walk model, i.e. that the coverage of Ag on the large particle surface is low. This guarantees that the system is far from saturation, and that Equation (S10) can be approximated by a linear behavior.

Table 1. Fitting parameters obtained from the data shown in Figure 7. The 'maximum coverage' parameter shows the maximum fraction of the surface of the large particle covered by Ag over the experimental concentration range, assuming that this value follows a Langmuir isotherm dependence over $N_{A g}$. Some representative values of the dissociation constants $K_{D}$ of these ligandreceptor systems are also reported for comparison.

\begin{tabular}{|c|c|c|c|c|c|c|}
\hline System & $\alpha /$ molecule $^{-1}$ & $\begin{array}{c}1 / \alpha / \\
\text { molecule }\end{array}$ & $\beta$ & $\gamma$ & $\begin{array}{c}\text { Maximum } \\
\text { coverage }\end{array}$ & $\boldsymbol{K}_{\boldsymbol{D}} / \mathbf{M}$ \\
\hline $\begin{array}{c}\text { Biotin/ } \\
\text { streptavidin } \\
\text { in FBS }\end{array}$ & $1.3 \times 10^{-12}$ & $7.7 \times 10^{11}$ & $-1.5 \times 10^{-1}$ & 2.6 & $2.5 \times 10^{-3}$ & $4 \times 10^{-14}$ \\
\hline $\begin{array}{c}\text { TNF- } \alpha \text { in } \\
\text { FBS }\end{array}$ & $3.6 \times 10^{-14}$ & $2.8 \times 10^{13}$ & $-1.6 \times 10^{-1}$ & $1.3 \times 10^{-1}$ & $6.4 \times 10^{-4}$ & $\begin{array}{c}2.2 \times 10^{-9}-7.8 \times 10^{-9} \\
\text { from ref. }^{41}\end{array}$ \\
\hline
\end{tabular}

Comparison with other magnetic particle-based systems. Several recent reports have highlighted the capabilities of magnetic micro- and nanoparticles as carriers of the Ag or as detection labels for sensitive analysis. ${ }^{19,20,42}$ In a first class of devices, the Ag are initially bound to the surface of a microchannel, and are then detected by injecting Ab-labeled particles ('Ag on substrate', see Figure 8). Other devices, like the one discussed here, present the specificity of tethering the $\mathrm{Ag}$ of interest on the particles and bringing the latter to the detection area by a 
microfluidic flow ('Ag on particles'). First of all, both approaches of these so-called 'surface coverage' assays have resulted in an extremely low limit of detection. ${ }^{20}$ However, by comparing the dose-response curves, obtained over similar ranges of concentrations for these two types of devices ('Ag on substrate' vs 'Ag on particles'), different behaviors have been observed.

In the 'Ag on substrate' setup (see Figure 8), the Ag is bound to the bottom of a channel. Here, the detection process can be reduced to particles scanning linearly the surface of the channel, until they encounter an Ag. At low Ag concentrations, if enough particles have passed in the channel, so that all the adsorbed $\mathrm{Ag}$ are detected, the output is the amount of particles adsorbed onto the surface. As a consequence, the calibration curve is an adsorption isotherm, such as the Langmuir Equation described on Equation (S14). Interestingly, for this type of device, the concentration response drawn over a logarithmic scale typically is a sigmoid saturating at high concentrations. ${ }^{43,44}$ We note that a sigmoid is a good approximation of a Langmuir isotherm on a logarithmic scale. The flattening of the curve typically limits the capabilities of these chips at very high concentrations. For a Langmuir adsorption isotherm, this saturation theoretically occurs for concentrations larger than $1 / \alpha$ ( $\alpha$ where is the Langmuir constant from Equation (S14)). It is important to note that the $1 / \alpha$ parameter is actually setup-dependent. As seen from the curves shown in ref. ${ }^{43,44}$, the $1 / \alpha$ value is quite low in this type of devices (below $10^{10} \mathrm{Ag}$ ). The plateau, on the right side of the sigmoid, can be observed on the same range of concentrations considered in our device, indicating concentrations above the $1 / \alpha$ limit. However, for comparable ranges of concentrations, the 'Ag on particles' setup is not saturating, even at the highest concentration considered, which are still below their $1 / \alpha$ limit (see Table 1). This phenomenon is beneficial for widening the dynamic range of the device, and stems from the sub-saturation coverage of the $\mathrm{Ag}$ on the large magnetic particles. The regime of this device is therefore sub-linear, but saturation of 
the output will nevertheless occur if $N_{A g}$ exceeds $1 / \alpha$. Furthermore, the particle surface scanning process, described extensively before, is expected to improve the behavior of the device especially at low Ag surface coverage, as indicated by the graphical representation of $P_{\text {capture }}$ (Figure $4 \mathrm{f}$ ), and by the comparison of Equation (S1) and (S6).

It is important to notice that, for both geometries, the dose-response curves are expected to flatten for extremely high concentrations. This can be due to saturation of the binding sites or steric hindrance effects and is taken into account by the adsorption isotherm, present in the two different designs, describing the binding of the free Ag to their substrate. Also, in this high concentrationlimit, steric hindrance related to the size of the large particle will comparatively limit the efficiency of substrate binding as, above a certain Ag concentration on the surface of a large particle, not all Ags can contribute in improving the binding efficiency of the particle.

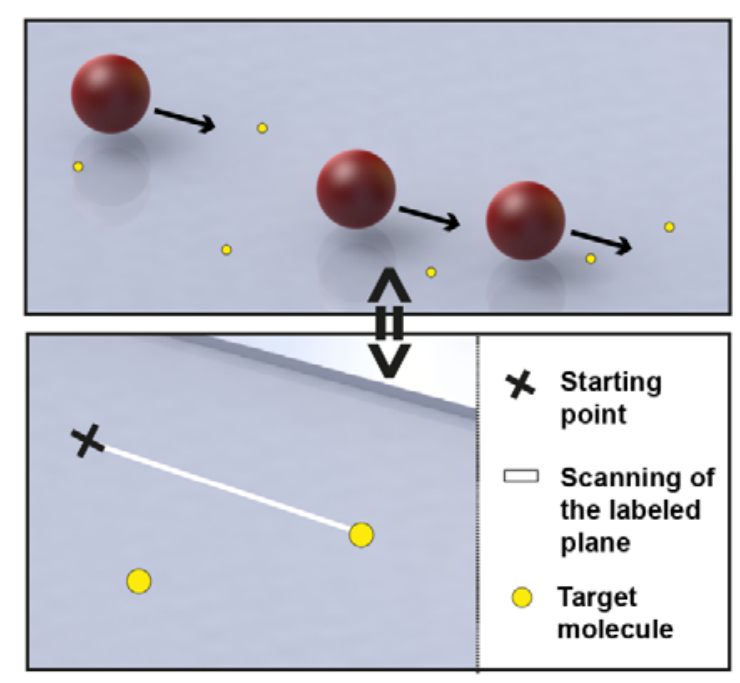

Figure 8. Principle of the 'Ag on substrate' magnetic particle-based system studied in literature: the target $\mathrm{Ag}$ molecules are bound on the substrate and $\mathrm{Ab}$-functionalized large particles scan the 
surface for detection. Counting of the immobilized particles quantifies the number of ligandreceptor bindings and hence the Ag concentration on the surface.

\section{CONCLUSIONS}

We have investigated the principle of magnetic particle-scanning for on-chip detection of biomolecules in a custom-built magneto-microfluidic chip and theoretically described the process by a random walk model. The latter explained the low limit of detection and the wide detection range of the experimental dose-response curves obtained previously for two different ligandreceptor systems (biotin/streptavidin and TNF- $\alpha$ ). ${ }^{18}$ Our model showed that magnetic particlescanning results in a very high probability of immunocomplex formation for very low $\mathrm{Ag}$ concentrations, which directly translates in an extremely low limit of detection, down to the single molecule-per-particle level. On the other hand, for high Ag concentration the binding efficiency is lowered, which is key to the large dynamic range of the dose-response curves. Moreover, our model allowed better understanding of the behavior of other types of particle-based heterogeneous immunoassays, which also exhibited very low limits of detection. We therefore think that, despite the specific nature of the magnetic particle scanning principle we proposed, our analysis has wider application potential and will be helpful for designing and explaining new types of on-chip surface coverage immunoassays, which exploit the binding of a particle to a substrate via an Ag.

\section{ASSOCIATED CONTENT}

Supporting Information. Additional material and details concerning theoretical modeling, experimental protocols and results are available free of charge via the Internet at http://pubs.acs.org. 


\section{AUTHOR INFORMATION}

\section{Corresponding Author}

* Author to whom correspondence should be addressed. E-mail: martin.gijs@epfl.ch

\section{Present Addresses}

†present address: Stanford University School of Medicine, Canary Center Early Cancer Detection, Stanford, CA 94305-5101, USA.

\section{Author Contributions}

The manuscript was written through contributions of all authors. All authors have given approval to the final version of the manuscript.

\section{ACKNOWLEDGMENT}

Funding of this work was provided by the EPFL, the EU Ideas program (ERC-2012-AdG-320404) and the EU People program (Marie Curie Initial Training Network 'Biomax', Grant No. 264737). The authors thank A. Sayah for discussion and help with optical microscopy and the staff of the Center of Micro- and Nanotechnology of EPFL for assistance in the microfabrication processes.

\section{REFERENCES}

(1) Etzioni, R.; Urban, N.; Ramsey, S.; McIntosh, M.; Schwartz, S.; Reid, B.; Radich, J.; Anderson, G.; Hartwell, L. Nat Rev Cancer 2003, 3, 243-252.

(2) Thaxton, C. S.; Elghanian, R.; Thomas, A. D.; Stoeva, S. I.; Lee, J. S.; Smith, N. D.; Schaeffer, A. J.; Klocker, H.; Horninger, W.; Bartsch, G.; Mirkin, C. A. P Natl Acad Sci USA 2009, 106, 18437-18442.

(3) Etzioni, R.; Kooperberg, C.; Pepe, M.; Smith, R.; Gann, P. H. Biostatistics 2003, 4, 523-538.

(4) Srinivas, P. R.; Kramer, B. S.; Srivastava, S. Lancet Oncol 2001, 2, 698-704.

(5) Barletta, J. M.; Edelman, D. C.; Constantine, N. T. Am J Clin Pathol 2004, 122, 20-27.

(6) de Jong, D.; Kremer, B. P.; Olde Rikkert, M. G.; Verbeek, M. M. Clin Chem Lab Med 2007, $45,1421-1434$.

(7) Galasko, D. J Alzheimers Dis 2005, 8, 339-346. 
(8) Giljohann, D. A.; Mirkin, C. A. Nature 2009, 462, 461-464.

(9) Anderson, N. L.; Anderson, N. G. Mol Cell Proteomics 2002, 1, 845-867.

(10) Chen, R. J.; Bangsaruntip, S.; Drouvalakis, K. A.; Kam, N. W.; Shim, M.; Li, Y.; Kim, W.; Utz, P. J.; Dai, H. Proc Natl Acad Sci U S A 2003, 100, 4984-4989.

(11) Gaster, R. S.; Hall, D. A.; Nielsen, C. H.; Osterfeld, S. J.; Yu, H.; Mach, K. E.; Wilson, R. J.; Murmann, B.; Liao, J. C.; Gambhir, S. S.; Wang, S. X. Nat Med 2009, 15, 1327-U1130.

(12) Krishnan, S.; Mani, V.; Wasalathanthri, D.; Kumar, C. V.; Rusling, J. F. Angew Chem Int Edit 2011, 50, 1175-1178.

(13) Morozov, V. N.; Groves, S.; Turell, M. J.; Bailey, C. J Am Chem Soc 2007, 129, 12628-+.

(14) Mulvaney, S. P.; Myers, K. M.; Sheehan, P. E.; Whitman, L. J. Biosens Bioelectron 2009, 24, $1109-1115$.

(15) Nam, J. M.; Thaxton, C. S.; Mirkin, C. A. Science 2003, 301, 1884-1886.

(16) Rissin, D. M.; Kan, C. W.; Campbell, T. G.; Howes, S. C.; Fournier, D. R.; Song, L.; Piech, T.; Patel, P. P.; Chang, L.; Rivnak, A. J.; Ferrell, E. P.; Randall, J. D.; Provuncher, G. K.; Walt, D. R.; Duffy, D. C. Nat Biotechnol 2010, 28, 595-U525.

(17) Zheng, G. F.; Patolsky, F.; Cui, Y.; Wang, W. U.; Lieber, C. M. Nat Biotechnol 2005, 23, 1294-1301.

(18) Tekin, H. C.; Cornaglia, M.; Gijs, M. A. Lab Chip 2013, 13, 1053-1059.

(19) Gijs, M. A.; Lacharme, F.; Lehmann, U. Chem Rev 2010, 110, 1518-1563.

(20) Tekin, H. C.; Gijs, M. A. M. Lab Chip 2013, 13, 4711-4739.

(21) Choi, J. W.; Oh, K. W.; Thomas, J. H.; Heineman, W. R.; Halsall, H. B.; Nevin, J. H.; Helmicki, A. J.; Henderson, H. T.; Ahn, C. H. Lab Chip 2002, 2, 27-30.

(22) Hayes, M. A.; Polson, N. A.; Garcia, A. A. Langmuir 2001, 17, 2866-2871.

(23) Lacharme, F.; Vandevyver, C.; Gijs, M. A. M. Anal Chem 2008, 80, 2905-2910.

(24) Peyman, S. A.; Iles, A.; Pamme, N. Lab Chip 2009, 9, 3110-3117.

(25) Lehmann, U.; Vandevyver, C.; Parashar, V. K.; Gijs, M. A. M. Angew Chem Int Edit 2006, $45,3062-3067$.

(26) Shikida, M.; Takayanagi, K.; Honda, H.; Ito, H.; Sato, K. J Micromech Microeng 2006, 16, $1875-1883$.

(27) Rissin, D. M.; Walt, D. R. Nano Lett 2006, 6, 520-523.

(28) Narhi, L. O.; Arakawa, T. Biochem Bioph Res Co 1987, 147, 740-746.

(29) Baudry, J.; Rouzeau, C.; Goubault, C.; Robic, C.; Cohen-Tannoudji, L.; Koenig, A.; Bertrand, E.; Bibette, J. P Natl Acad Sci USA 2006, 103, 16076-16078.

(30) Ranzoni, A.; Sabatte, G.; van IJzendoorn, L. J.; Prins, M. W. J. Acs Nano 2012, 6, 3134-3141.

(31) Zaouk, R.; Park, B. Y.; Madou, M. J. Methods mol biol 2006, 321, 5-15.

(32) Fonnum, G.; Johansson, C.; Molteberg, A.; Morup, S.; Aksnes, E. J Magn Magn Mater 2005, 293, 41-47.

(33) Chemla, Y. R.; Crossman, H. L.; Poon, Y.; McDermott, R.; Stevens, R.; Alper, M. D.; Clarke, J. P Natl Acad Sci USA 2000, 97, 14268-14272.

(34) Bruus, H. Theoretical Microfluidics; Oxford Univ Press: New York, 2008.

(35) Cornaglia, M.; Tekin, H. C.; Lehnert, T.; Gijs, M. A. M. J Appl Phys 2013, 114.

(36) Morozov, V. N.; Morozova, T. Y. Anal Chim Acta 2006, 564, 40-52.

(37) Choi, S. Y.; Karp, J. M.; Karnik, R. Lab Chip 2012, 12, 1427-1430.

(38) Sivagnanam, V.; Sayah, A.; Vandevyver, C.; Gijs, M. A. M. Sensor Actuat B-Chem 2008, 132, 361-367. 
(39) Lee, N. K.; Johner, A.; Thalmann, F.; Cohen-Tannoudji, L.; Bertrand, E.; Baudry, J.; Bibette, J.; Marques, C. M. Langmuir 2008, 24, 1296-1307.

(40) Holmberg, A.; Blomstergren, A.; Nord, O.; Lukacs, M.; Lundeberg, J.; Uhlen, M. Electrophoresis 2005, 26, 501-510.

(41) Chiu, W. C.; Lai, Y. P.; Chou, M. Y. Plos One 2011, 6.

(42) Gijs, M. A. M. Microfluid Nanofluid 2004, 1, 22-40.

(43) Shlyapnikov, Y. M.; Shlyapnikova, E. A.; Simonova, M. A.; Shepelyakovskaya, A. O.; Brovko, F. A.; Komaleva, R. L.; Grishin, E. V.; Morozov, V. N. Anal Chem 2012, 84, 5596-5603. (44) Tsai, H. Y.; Hsieh, Y. C.; Su, Y. M.; Chan, J. R.; Chang, Y. C.; Fuh, C. B. Biosens Bioelectron 2011, $28,38-43$. 
For Table of Contents Only:

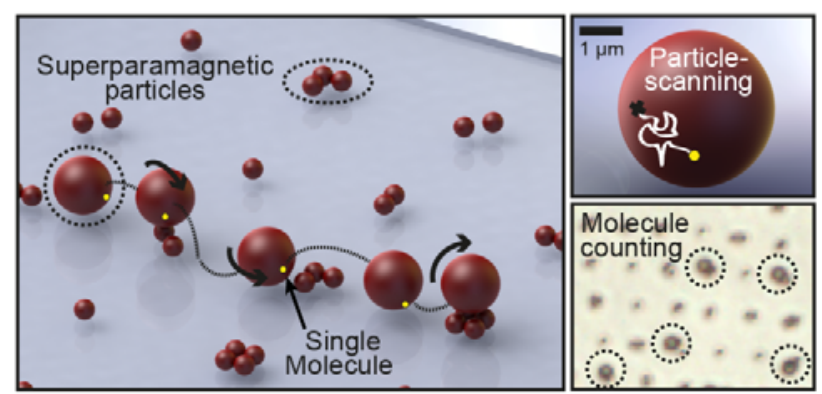

